\title{
EUCLID RTP 8.2 IRST Software Simulator
}

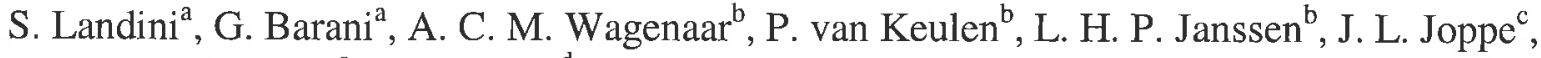 \\ P. B. W. Schwering ${ }^{c}$, C. Visconti ${ }^{\mathrm{d}}$ \\ aALENIA DIFESA Unita' Officine Galileo, via A. Einstein 35, 50013 Campi Bisenzio, Italy \\ ${ }^{b}$ Hollandse Signaalapparaten B. V., Zuidelijke Havenweg 40, 7554 RR Hengelo, The \\ Netherlands \\ 'TNO-FEL, Oude Waalsdorperweg 63 P. O. Box 96864, 2509 JG The Hague, \\ The Netherlands \\ ${ }^{\mathrm{d}}$ SAGEM SA, 72-74 rue de la Tour Billy, BP72, 95101 Argentuil CEDEX, France
}

\begin{abstract}
Within the RTP 8.2 one of the main goals of the START programme is to develop a software simulator which incorporates the results of the research activities devoted to evaluate new IRST technologies and technical solutions which can be the basis of new concepts for future generations IRSTs. The software simulator development is based on the use of a commercial framework to speed up its implementation. The main simulator features are: modularity, expandability, capability to simulate the IRST behaviour in different situation and under various scenarios. It is based on an analytical and statistical approach and provides an easy user interface with friendly tools for data supplying and results presentation
\end{abstract}

Keywords: Infrared Search and Track, simulator, framework, Figure Of Merit, performance flow, image flow

\section{INTRODUCTION}

The aim of the START (Search, Track And Recognise Threat) project, is to research system concepts and novel technologies for a future Infrared Search and Track (IRST) system which represents a considerable advance on current systems. Furthermore a simulator which incorporates the results of the research activities is in development.

The simulator envisaged by the START consortium is based on the use of a supporting FrameWork (FW) which simplifies the simulator software (SW) development and allows to expand the simulator as soon as new technologies and concepts become available.

Within the Simulator the system concepts are defined in a modular way and the IRST performances are evaluated through two chains: by a Figure of Merit/Performance flow and by an Image/Data flow to improve accuracy of calculated results and to check the chosen model, links between two chains have been conceived too.

Both parts have an input module in common, in which targets, scenario conditions and platforms are defined.

The system concepts make use of the technologies investigated in the project and modelled in the simulator. The technology areas taken into account are optics, scanning, detectors, processing and algorithms.

IRST system performance is evaluated within the simulator against defined Figures Of Merit (FOMs), and results from technology demonstrations carried out in test beds developed within the project can be inserted within the simulator. An output image obtained by the input image interaction with the system chain is supplied too.

\section{IRST SYSTEM CONCEPTS}

From the analysis of current IRST systems the START consortium proposed three concepts ${ }^{1}$ which give greater performance in terms of sensitivity, angular resolution, Field Of Regard (FOR) coverage and shorter update cycles.

The first concept, named "The Cupola" in the basic configuration consists of a dual band total staring concept with a Vertical Field Of View (VFOV) of $0^{\circ} \div 20^{\circ}$ and an Horizontal FOV (HFOV) of $180^{\circ}$ (figure 1).

This FOV will be covered by 9 Focal Plane Arrays (FPA) for the $3 \div 5 \mu \mathrm{m}$ band and 9 FPAs for the $8 \div 12 \mu \mathrm{m}$ band. So, each FPA will have a FOV of $20^{\circ} \times 20^{\circ}$, using single band optics. In the $3 \div 5 \mu \mathrm{m}$ band $(1024 \times 1024$ detector arrays) this results in an Instantaneous FOV (IFOV) of $0.34 \mathrm{mrad}$. The $8 \div 12 \mu \mathrm{m}$ band (consisting of $512 \times 512$ detector arrays) has 
a resolution of $0.68 \mathrm{mrad}$. Many options have been considered which concern either with an improved number of sensor and the use of spectral discrimination by a rotating optical filter.

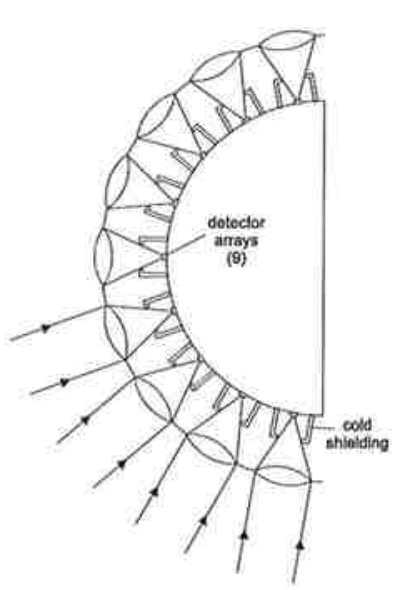

Figure 1: The Cupola concept schematic view
The second concept named Recognise While Scan (RWS) is an evolution of a classical IRST, as it is based on a scanning head which covers a FOR of $360^{\circ}$ with a bispectral channel $(3 \div 5 \mu \mathrm{m}$ and $8 \div 12 \mu \mathrm{m})$ with linear arrays with $1024 \times 4$ elements. The innovation of this solution consists in the capability to perform a foveal vision in a reduced region of the FOR by means a staring detector operating in the $3 \div 5 \mu \mathrm{m}$ spectral band, which carries on the recognition contemporaneously to the searching process.

The fact that the recognition can be provided many times for each single sweep of the FOR performed by the scanning head in about $400 \mathrm{~ms}(2.5 \mathrm{~Hz})$ is the real innovation of this concept. This is possible because a counter-rotating mirror allows to stop the relative motion of the scene during the scanning of the FOR.

A schematic view of this concept is represented in figure 2 .

The third concept is called the "MOSAIC" and is a distributed staring sensors system, consisting in six Search Heads ( $\mathrm{SH}$ ) and one Threat Recognise Head (TRH) as schematically represented in figure 3 .

For each sensor head, the FOR is obtained by moving one or two mirrors, but not through a scanning system, but in a "step-and-stare" operating mode.

The system FOR is then the juxtaposition of each sensor FOR, which is composed of several

instantaneous FOVs, thus drawing a mosaic. The period of the imaging system is given by the delay to see twice the same FOV on a given sensor head.

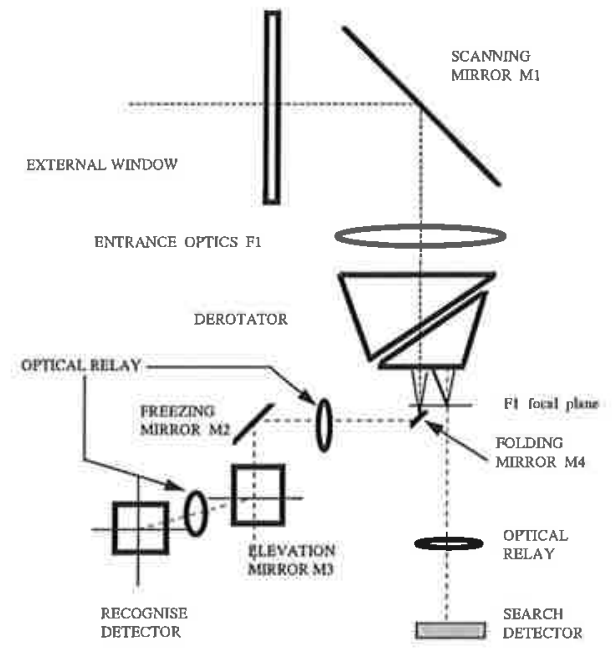

Figure 2: The RWS concept schematic view

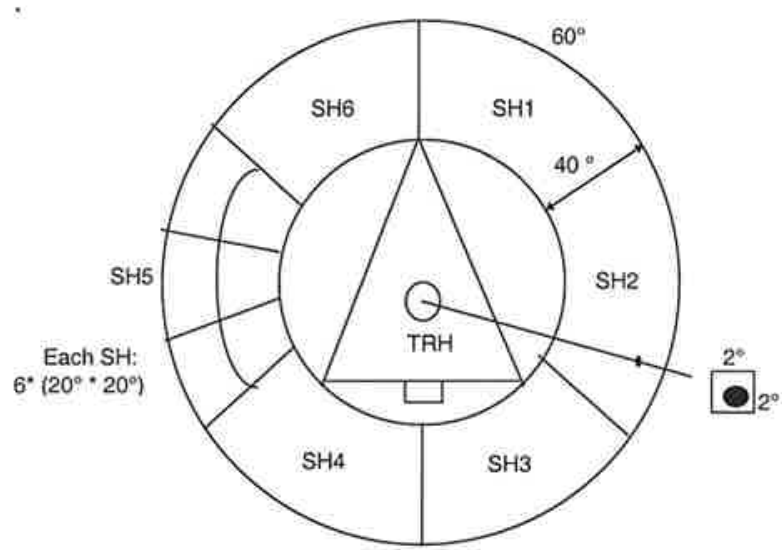

Figure 3: The Mosaic concept schematic view 
Each SH has a bispectral optics with a FOV of $20^{\circ} \times 20^{\circ}$ matched by a beam splitter to two staring arrays $1024 \times 1024$ $(3 \div 5 \mu \mathrm{m})$ and $512 \times 512(8 \div 12 \mu \mathrm{m})$. The TRH FOV is $2^{\circ} \times 2^{\circ}$ and work on the $3 \div 5$ band with a $1024 \times 1024$ staring array.

\section{SIMULATOR REQUIREMENTS AND GENERAL CONCEPTS}

\subsection{Requirements for the simulator}

In order to give concret form to the system concepts and solutions developed during the first years of the START programme the development of a SW simulator incorporating the results of the research activity has been foreseen: the simulator is the envisaged means by which all the efforts of the study performed by the project participants are integrated.

First of all a list of general requirements for the simulator have been set by the partners of the project: it must perform a global performance analysis of the simulated system in order to evaluate the global system design concepts and subsystem technologies. The simulator must evaluate the performance of each subsystem (optics, scanning, detector, processing) which constitutes the IRST, combining the results in order to assess the overall system performance.

The performance evaluation of the system design concepts and subsystem technologies must be carried out in various situations and under various scenarios. This capability of simulating IRST behaviour in different scenarios needs information on: weather, clutter, atmosphere, targets, sensor mission, potential non-target objects, platform behaviour. The simulator includes a reference system concept which represents the current IRST technology. The merits of new technology development and new system concepts can be evaluated by comparing its performance with the performance of the reference system for the same scenarios.

From the operational point of view the simulator must provide an easy user interface (masks which allow to select all the parameters characterising the simulated system and the environment: target, scenario, spectral bands, etc.), tools for data supply (input images or sequences from the database), tools for data/results presentation: easy comparison with settled performance requirements or performance of different system concepts/subsystem technologies.

It must consist in an analytical model with a partial image flow in order to support the analytical model. The simulator must provide a flexible structure to deal with the extraction of data from the image flow towards the analytical model. Modularity and extensibility are also requirements for the simulator: that is the possibility to join new modules, new system concepts and technologies

\subsection{Performance evaluation approach}

The methods commonly used to simulate image processing systems in order to evaluate and compare their performances can be roughly classified as analytical, statistical or hybrid.

\section{Analytical approach}

In the first case the items which flow along the simulated chain are numbers, being values of FOMs. For each stage of the system the relationship between input and output is established via a mathematical model of the module. Creating these relationships provides a good insight in what causes an IRST to perform. Another advantage is the possibility to look for analytical solutions for the tuning problem: simple optimisation and assessment. A disadvantage is the fact that the assumptions on which the relationships are based could be disputable or at least limit the domain of applicability of the analytical relationship.

\section{Statistical approach}

In the second case the items flowing along the chain are digital images (sequence of digitised frames) or numerical information extracted from each image (which is processed by the algorithmic part of the system). Thus simulating the system response on a large number of images allows to derive statistical results on the global behaviour of the system. The relationship between input and output is established using statistical tools. In this case the method is straightforward, no assumptions or limitations are required, but the disadvantage is that it requires far more processing capacity for numerous Monte Carlo runs, i. e. heavy data processing.

\section{Hybrid approach}

In the third approach for each performance module an analytical surface is fitted to a limited set of empirical performance results obtained with real or simulated imagery, in order to establish the connections between the input and the output of the module. The main advantage is that the method does not require heavy data processing and it is modular. Drawbacks are the complexity of the functional description of the simulator, the sensitivity to the specific scenarios, to the targets characteristics and also the number of iterations runs requested to establish the surface.

\section{Selected approach}


The chosen structure of the simulator we are implementing is the hybrid one containing elements taken from both the analytical and statistical approaches. It consists of an analytical model with a partial image flow: the first chain (performance flow) contains the analytical description of the subsystem performances (analytical approach).The FOMs and the relationships among them are taken from the literature, from worker's experience or from the technology work elements within the RTP 8.2.

The second chain materialises the statistical approach by implementing a partial (not every stage is included) data flow: the data are images or, in the post-processing part after the detection module, information taken from the processed images (ROIs, segments, centroids locations etc.).

In order to make the IRST performances evaluation more accurate and realistic the links between the outputs (the measured performances) of the stages in the data flow chain (statistical results) are performed not through an analytical surface but through empirical rules called "rules of thumb" (RoTs).

The RoT are the resulting transfer functions of the performance flow chain obtained through the empirical results of the data flow chain. They are similar to the rules of the expert systems which derive from an encoding of the experiences and allow to infer conclusions from empirical or simulated data flow observations. The RoTs (simple formulas) are derived by separately studying the parts of the system they are implemented in: the limits of validity for their application may be narrow and must be specified.

As an example of a RoT consider the performances of the mechanical stabilisation using Inertial Navigation System (INS), which is part of the stabilisation performance module:

$$
\delta \alpha \leq 5 \cdot \delta_{\text {INS }}+0.5 \cdot \text { IFOV }
$$

where:

$\delta_{\text {INS }}: \quad$ error of INS system (input)

IFOV: instantaneous FOV (input)

$\delta \alpha: \quad$ angular error (output)

A new and important advantage of this approach is to provide the possibility to insert results from technology test-beds, that is partial technology demonstrations carried out externally to the SW simulator through hardware implementation of submodules concerning optics, scanning, detectors, processing and algorithms.

\subsection{System Performance Model (SPM)}

The System Performance Model (SPM) implements the simulator performance flow chain and constitutes the kernel of the simulator. This is depicted in figure 4

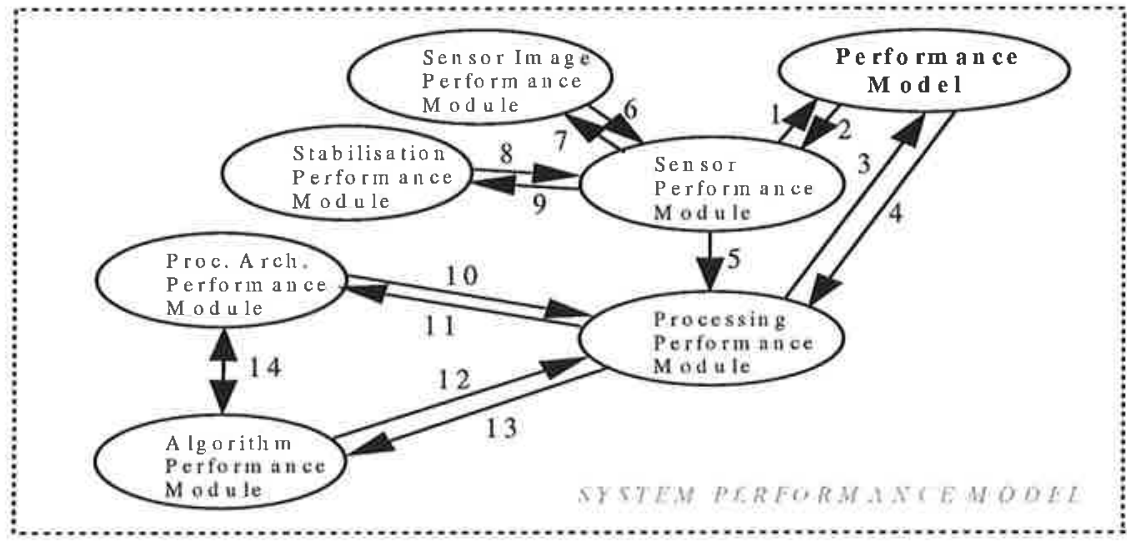

Figure 4: Scheme of the System Performance model

The SPM consists of the Sensor Performance Module (SnPM) and Processing Performance Module (PPM).

From the input data, which define the scenario and describe the architecture and parameters provided by two modules external to it (the Scenario Definition Module and the System Concept Module, described below), the SnPM computes the performance (values of FOMs) of the IRST. It models the hardware like the sensor head and the data (image) transmission devices. It also includes all devices used for stabilisation and directing the camera head.

The involved technologies are: optics, scanning, detector.

The PPM includes the digital processing hardware and the algorithm performance calculations. The involved technologies are: processing and algorithms.

The SnPM consists of: Sensor Image Performance (SIP) Module and Stabilisation Performance (StP) Module

The PPM consists of: Processing Architecture Performance (PAP) Module and Algorithm Performance (AP) Module. 
In order to compute the system performances in terms of FOMs values the Performance Model (PM) calls the Sensor Performance Module and the Processing Performance Module. The PM provides them (links 2 and 4) with the information concerning the requirements, that is which FOMs have to be calculated. The selection of particular FOMs at the output of the PM imposes to compute other particular FOMs by the SnPM and PPM. The parameters are provided to them by the System Concept Module (this auxiliary module is explained below). The PM collects the results from the SnPM and PPM (links 1 and 3) and compute the required global FOMs values by joining their contributes.

Link 5 shows the data flow from the SnPM to the PPM about the scene information, target and background characteristics (target information and background characteristics).

Analogously the SnPM calls the SIP and the StP sub-modules and the PPM call PAP and AP sub-modules: the submodules are provided with the requirements information to compute the right FOMs (links 7, 911 and 13), the parameters are provided by the System Concept Module. By collecting the data (FOM values) coming from the submodules (links 6, 8, 10 and 12), they compute the FOMs related to the Sensor and Processing Modules.

Link 14 represents the exchange of data between the PAP and AP modules.

The SIP, StP and AP modules are singularly related with the partial data flow (statistical chain external to the SPM) to make the results more accurate and realistic (use of RoTs).

The four technology modules (SIP, StP, PAP and AP) just mentioned, base their output on the system concept parameters, which are received from the System Concept Definition Module.

Actually the project goal is to compute the following set of output FOMs for a given, specific system in an also specific scenario:

- Probability of target detection

- Probability of target track handover

- Probability of alert on the target

- Range

- Accuracy of elevation and azimuth

- False Detection Rate

- False Track Rate

- False Alert Rate

- Effective target resolvability

- Capability of the system (max. \# tracks)

The simulator can also be used for performance comparison of different systems and for performance comparison of different interchangeable technologies within a given system.

\subsection{Simulator auxiliary parts}

Every function that the SPM needs is carried out by an auxiliary simulator module. The following operations and the corresponding modules have been envisaged as auxiliary simulator functions:

- Insertion of the information about the functions of the simulator (that is the FOMs we want to compute) and the performances, in terms of FOM values, to be obtained. These information can be in form of requirements to the simulator and are stored into the System Requirement List (SRL).

Besides, it will be possible to insert weights in order to express the importance the user assigns to each output FOM and to assign weights associated with different input scenarios (in order to give more importance to the scenarios the user is more interested in). The purpose is to have an unique global system score which in addition to the output FOM values, allow to compare different system architectures/concepts (see the Trade-off module below).

The simulator provides the Man Machine Interface (MMI) to manipulate the FOMs and scenario weights.

- The second required functionality is the capability of supplying the necessary inputs: image representing real or synthetic scenes for the data flow chain, information characterising the signal, the clutter inside the images (SCR and Clutter) for the performance flow chain. The modules supplying these data are the Scenario Definition \& Generation Modules (SDM \& SGM). The information characterising the scenarios (scenario definition data, i.e. target size, aspect angle, contrast in terms of SNR, background clutter statistics, atmospheric features) are stored into files external to the simulator.

- As the IRST system is conceptually split into functional subsystems (optics, scanning, detection, processing, ...), the system concept defines how these subsystems work together, that is the global system architecture. The values of the parameters involved into each subsystem are related to the adopted technologies to implement it.

All system concepts will be completely described by the System Concept Module, combined with a set of parameters which can be varied to obtain slightly different systems. Parameters value sets will be recorded in System Concepts Description Files, which specify the parameters set for each subsystem. The simulator provides the MMI tools to manipulate them. Further, it selects the system architecture and the parameters for each module. A reference system 
concept obtained considering a classical structure with parameters describing a typical modern system has been inserted in the simulator.

- After the FOMs have been calculated, we need to compare them with the results of previous trials, obtained from different input parameters, different technologies or different system concepts. In order to get this comparison faster the aim is to compute an unique global score.

Our approach is to allow the user to insert into the Simulator the weighting factors by multiplying the SPM resulting FOMs in order to get an unique score. In this way different users can assign different scores to the importance of the output FOMs, increasing the weights of the FOMs they are more interested in.

The same approach is followed to evaluate the influence of the different scenarios: the score obtained with a particular scenario is weighted with a factor inserted by the user, specifying the user interest.

The modules in charge of computing the global system scores are the Trade-Off Model \& FOM Weighting Module.

One of the most interesting advantage of the global score computation is the possibility to automatise an optimisation process. This automatic optimisation process can be performed through a "linkage between the calculation of the system parameters and the parameter iteration" ${ }^{\prime 3}$. The output FOMs (and global score) are computed iteratively for values of the input parameters which are scanned on predefined ranges, the best solution (maximum score) and the corresponding parameters values can be automatically selected.

The FOM weighting module uses weights derived from the SRL and performs for each FOM the translation from the FOM value into the final score. This module allow to insert different weights values for different users. Each user can define his own FOMs to compare different system concepts against his own criteria.

In figure 5 the Operational Simulator Block Diagram (OSBD) is shown, it is a schematic representation of the simulator from the operational point of view. In the figure the main operative modules constituting the simulator, the data flow chain and the performance flow are outlined.

The data flow chain works iteratively, with a multiplicity of operative situations concerning the type of scenario (specified in the SDM), and with more system concepts specifications (specifying the system structure and parameters into System Concepts Description files). Results are the selected FOMs at the outputs of all the blocks simulating the IRST system. They are inputs to the statistic module whose results allow to adjust the parameters for the SPM (RoT).The trade-off module presents the results and can translate these performance figures into an unique performance score using the obtained FOMs and the FOM weightings.

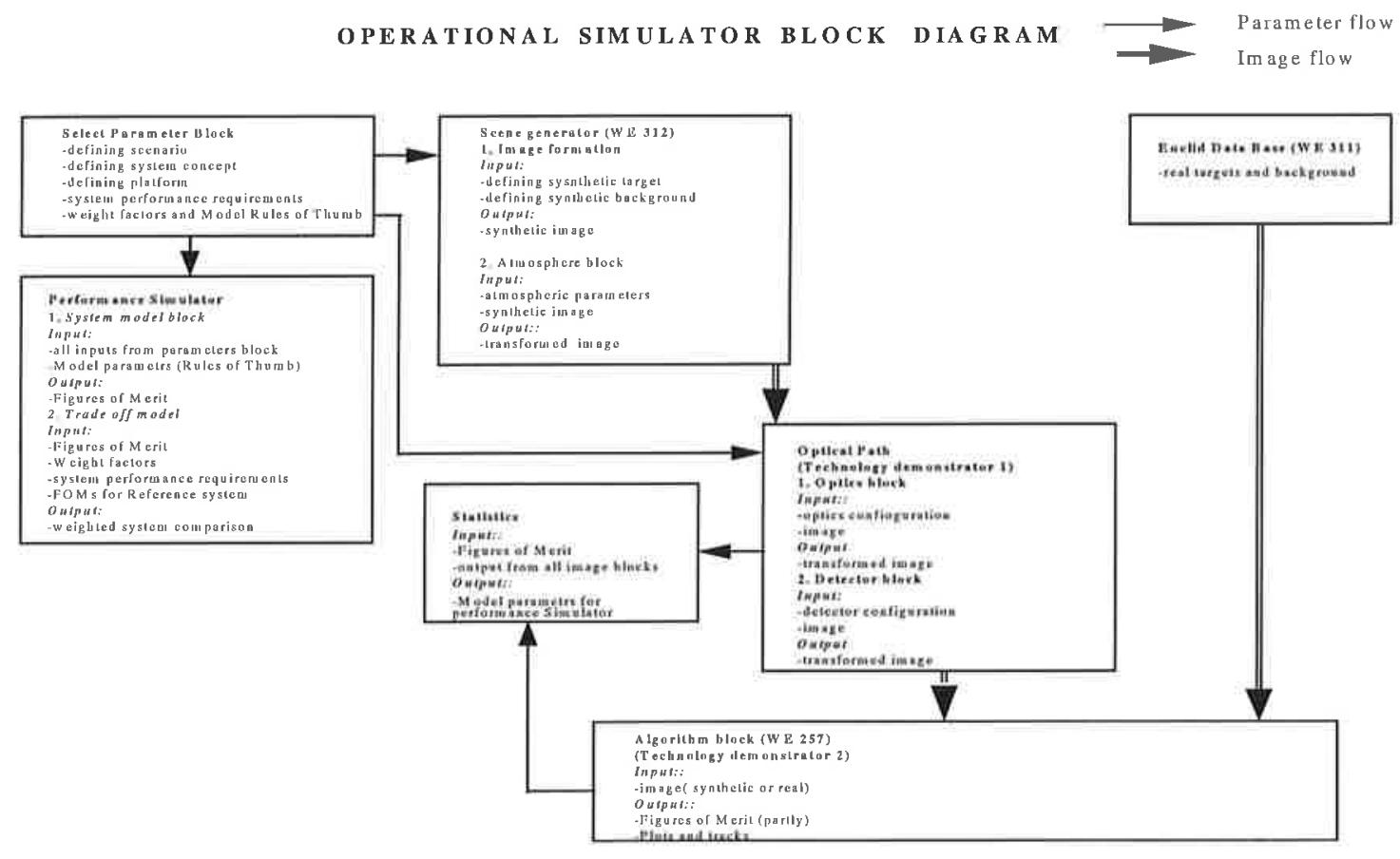

Figure 5: View of the Operational Simulator Block Diagram (OSBD)

\section{SIMULATOR MODULES}


The scene is generated through different modules for the target, background and the intermediate atmosphere. These separate issues are combined for generating a scene sequence for the data flow part of the simulator. The information for the scene generation is taken from the Scenario definition module: the scenario definition files contain information concerning targets features (type, size, aspect angle, contrast), background features (background clutter statistics) and atmospheric features. The generated image sequences are independent from the actual sensor parameters (even if sensor parameters are needed on the input to limit the amount of calculations). They are based on the spatial resolution of the sensor (minimum of approximately $2 \times 2$ meter, the maximum is based on the IFOV/PSF of the sensor system), and wave bands. Only broad band scenes are generated, one in the $3 \div 5$ and the other in the $8 \div 12$ micron band.

The scene 'pixel' based insertion procedure will be as follows:

$$
\mathrm{PATH}_{v}+\tau_{v}\left(\mathrm{CVTAR} v+(1-\mathrm{CV}) \mathrm{BG}_{v}\right)
$$

where

$\begin{array}{ll}\mathrm{CV}: & \text { scene pixel coverage fraction of the target from the target module. } \\ \mathrm{BG}: & \text { the local background radiance from the background module. } \\ \mathrm{PATH}: & \text { the path radiance defined by }(1-\tau) \mathrm{BB}\left(\mathrm{T}_{\text {air }}\right) \\ \mathrm{BB}\left(\mathrm{T}_{\mathrm{air}}\right): & \text { path atmospheric radiance } \\ \mathrm{T}_{\text {air }}: & \text { atmospheric temperature } \\ \tau: & \text { the transmission from the atmosphere module. } \\ \mathrm{TAR}: & \text { the target radiance from the target module. } \\ v: & \left.\text { the spectral wave band parameter (wave number } \mathrm{cm}^{-1}\right) .\end{array}$

The combined effect of target radiance and coverage factor are the target radiance on the scene 'pixel' (Watt $\left.\mathrm{m}^{-2} \mathrm{sr}^{-1}\right)$. Other required input parameters for the scene sequences generation are: image size (e.g. power of two in pixels) in meters, temporal resolution in seconds (frame generation interval), sequence length in seconds, sensor position and looking direction. The output consists of scene sequences in radiance.

Concerning the performance flow chain, output data need to be characterised in general terms generated in the target area with respect to the local background: Signal to Clutter Ratio (SCR), integrated Contrast Target Signal (CTS), Background Clutter in terms of Clutter to Noise Ratio (CNR), target Minimum bounding BOX (MBOX), effective Target SiZe (TSZ).

Generated target types are: air targets (aeroplane), land target (tank), symbolic targets (point source, sphere, shoebox). The targets are modelled from a 3D geometrical and temperature description, a 2D representation of these targets are available in the model. Required input parameters are: target orientation, moving target path in the scene (distance, height, azimuth), insertion radiance factor (related to local signal-to-clutter ratio). Parameter data required for each selectable target are facet model and surface temperatures for each facet.

Concerning the atmosphere module: from MODTRAN transmission numbers are generated in a set of scenarios determined by Mid-Latitude Summer (MLS) and Mid-Latitude Winter (MLW) conditions, for long range horizontal paths. The transmission values are used for the generation of the atmospheric extinction.

Required input parameters are meteorological data (air temperature, background temperature, relative humidity, pressure, etc.), scenario information (sensor mission, platform path and target locations).

The background is characterised by average temperature, standard deviation and correlation coefficient in a two-fold "Itakura-like" procedure ${ }^{2}$. Temporal structure is created by taking completely correlated structure based on the background type parameters as a basis for the background radiance. Special clutter factors can be included in the scene to incorporate high clutter values. A separation of the scene in two areas, like the separation of a horizon, are foreseen. Required input parameters are background type (rural, urban, air), meteorological parameters (see atmosphere). Background is considered at the same distance as the target. Parameter data in the background model required for each background type are: average background temperature parameters/offsets (based on meteorological condition), constant standard deviation, constant correlation coefficient, variable standard deviation, variable correlation coefficient, clutter threshold and factor.

A limited selection of images can be used on-line from the START database of real imagery: they must be distinguished from the synthetic database because the same operation can not be carried out either on real and synthetic data. In principle no targets are inserted in this situation.

\subsection{System concepts module}


The main functional blocks which constitute a generic IRST system are represented in figure 6, such a scheme represents the Functional Block Diagram (FBD) of the system at low level of detail, it allows to identify the main modules and interfaces. Such FBD represents mainly Cupola and Mosaic concepts, while RWS has a slightly different diagram.

We define the technologies and the obtainable performances from each module by specifying the way each block can be built and the working parameters. The System concepts are defined by specifying the way the blocks are connected each other.

Many modules are conceptually the same for all the system concepts, but their models are characterised by different sets of parameters. The parameters characterising different system concepts are contained in different system concept files.

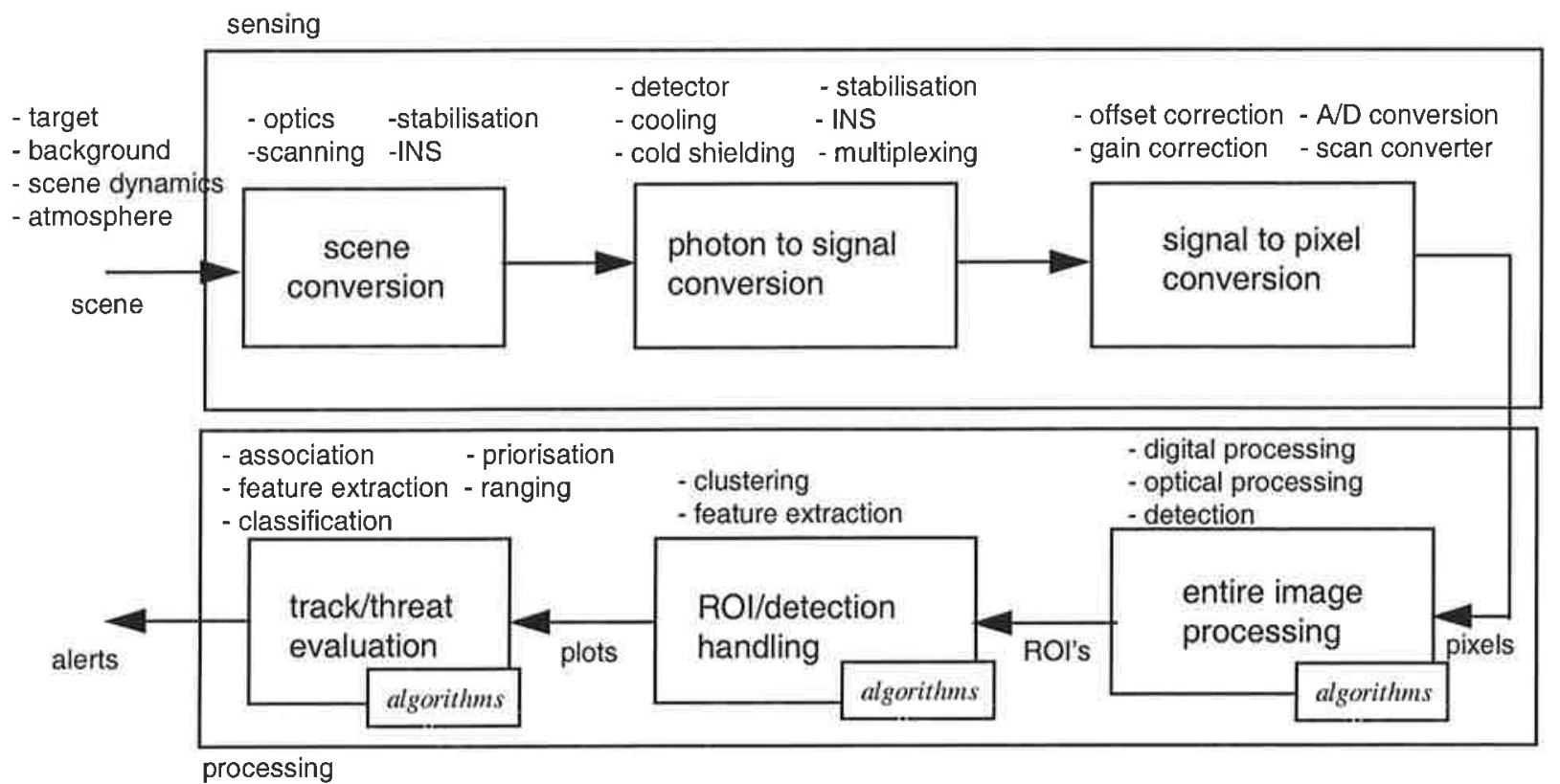

Figure 6: Generic IRST Functional Block Diagram (FBD)

\subsection{Scanning/optic module}

The module simulating the diffractive optics in the data flow chain works by convoluting the mask representing the optical PSF with the input image.

The aim of the scanning module in the RWS system concept is to evaluate the errors performed for the control of the search and recognition mirrors LOS and to estimate the errors carried out in the stabilisation process. These LOS stabilisation residuals will be further compensated through the "microscanning module" (elastic gimbals).

The simulation is constituted by a part describing the movement of the main objects generating errors (platform, rotation and elevation of M1 mirror, elevation of M3 mirror).

The second part of the simulation implements the needed algorithms in order to control the M1 and M3 mirrors which perform the recognition in the RWS system concept.

The last part of the simulation estimates the error performed in the LOS stabilisation process for the M1 and M3 mirrors and provides an estimation for the validity of this estimate.

In figure 7 the FBD of the scanning block for the RWS system concept is reported. 


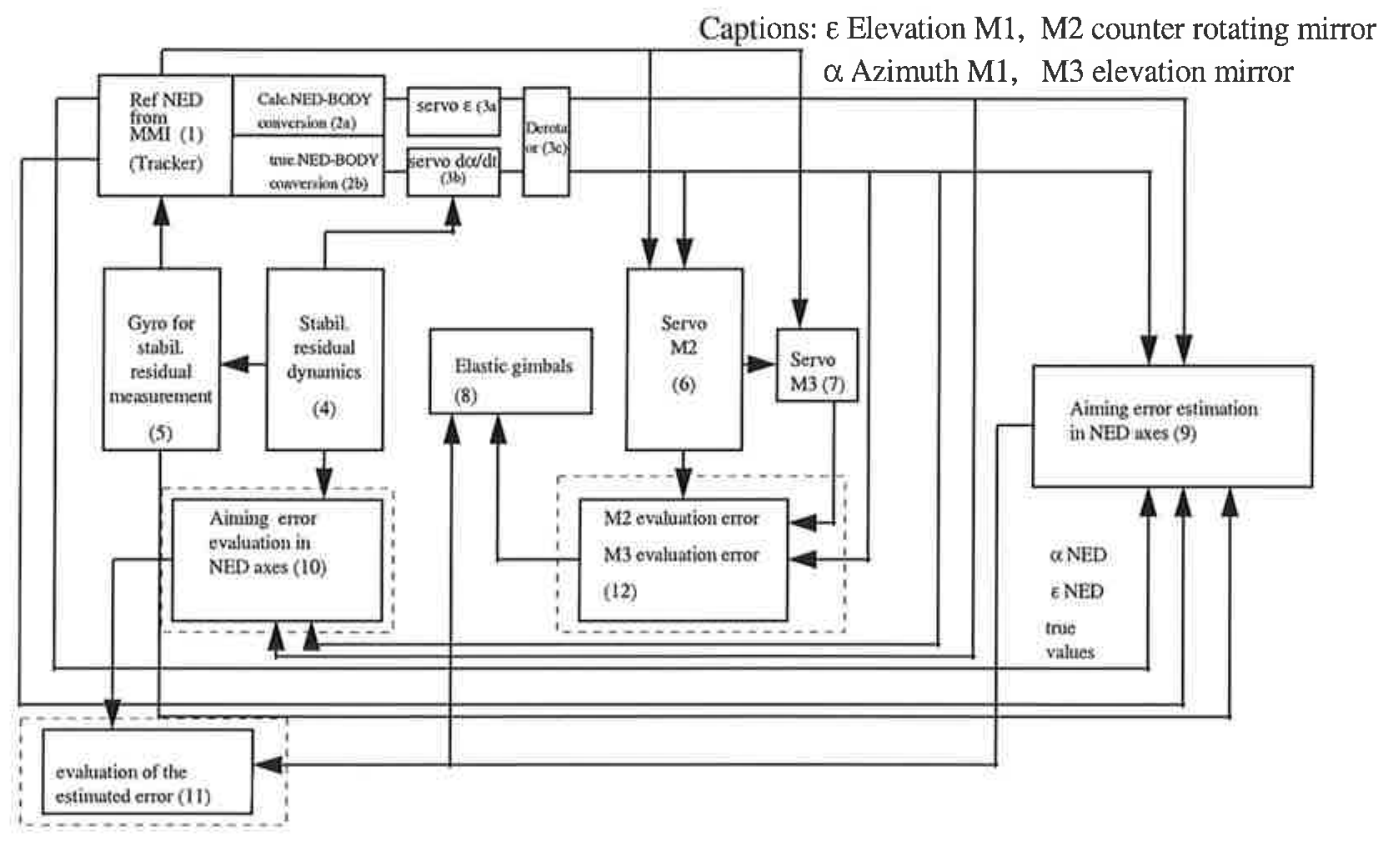

Figure 7: FBD of the scanning block of the RWS system

The sub-modules 1, 2 and 3 generate the command for the LOS stabilisation

The sub-modules 4 and 5 measure the platform stabilisation residuals

The sub-modules 6 and 7 describe the M1 and M3 movements

The sub-module 8 simulates the elastic gimbals behaviour

The sub-modules 9, 10 and 11 compute the LOS error estimate and evaluate the goodness of the estimate itself

The module 12 evaluates the error performed in the derotation process (in order to fix the image) and in the ROI selection

\subsection{Detector module}

This module generates the pixels response map taking into account the different solid angles and vignetting associated to each pixel due to optics and cold shield. Through the pixel response maps the flux impinging on each pixel is calculated and the generated charge is obtained as a function of integration time, detector response, pixel gain and offset. Different detector operating temperatures can be inserted so modifying the detector response, also fixed pattern noise and dead elements number can be introduced. Pixel output is digitised and a non uniformity correction is applied. The obtained image is compared with the input one in order to adjust the integration time which is used for the next frame acquisition (figure 8). Last NEI and NETD values for each pixel are calculated.
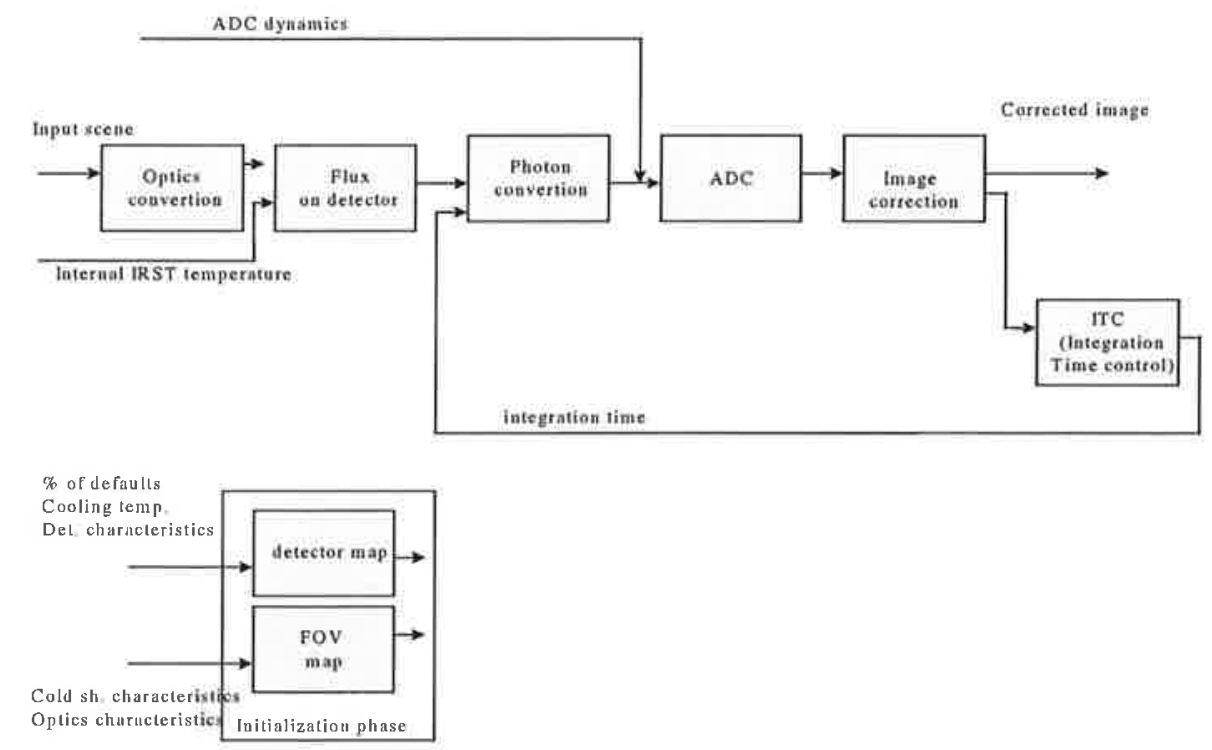

Figure 8: FBD of the optics-detector block 


\subsection{Algorithm module}

The module simulating the algorithms of the IRST system consist of the following blocks:

stabilisation,

detection of

$$
\begin{aligned}
& \text { stationary point targets } \\
& \text { extended targets } \\
& \text { moving targets } \\
& \text { tracking } \\
& \text { prioritisation, with } \\
& \text { recognition } \\
& \text { passive ranging }
\end{aligned}
$$

prioritisation, with

In figure 9 the algorithm combination diagram is shown with its interconnections. The input data consist of images created by the detector.

Stabilisation of the IRST image data is required in order to have spatially aligned information during the processing time scales.

This is achieved by deriving registration parameters from the image data by a Fourier Registration Algorithm and a Feature Tracking Algorithm. These algorithms make use of structure in the scene.

The obtained parameters are then applied in interpolation techniques to improve the stabilisation relative to the reference image.

Target detection is performed at three parallel stages for detection of stationary point targets, extended targets, moving targets.

Most of the effort in detection in EUCLID RTP 8.2 has gone in extended and moving targets. A number of extended detection algorithms have been pursued, such as region growing, feature extraction of hot blobs, extraction of edge and region features using a graph search engine, metric evaluation of grid cells. Moving target schemes are still being studied. These are Track Before Detect, Wavelet algorithms, algorithms based on region growing, target bounding, and feature tracking.

Detection of stationary point targets makes use of the earlier work on extended targets detection.

Plot data from the detection stages is used in the tracking. The approach leads to a track comparison between classical and state of the art techniques. The tracks are prioritised based on recognition parameters and passive range information. The output data consists of a track table.

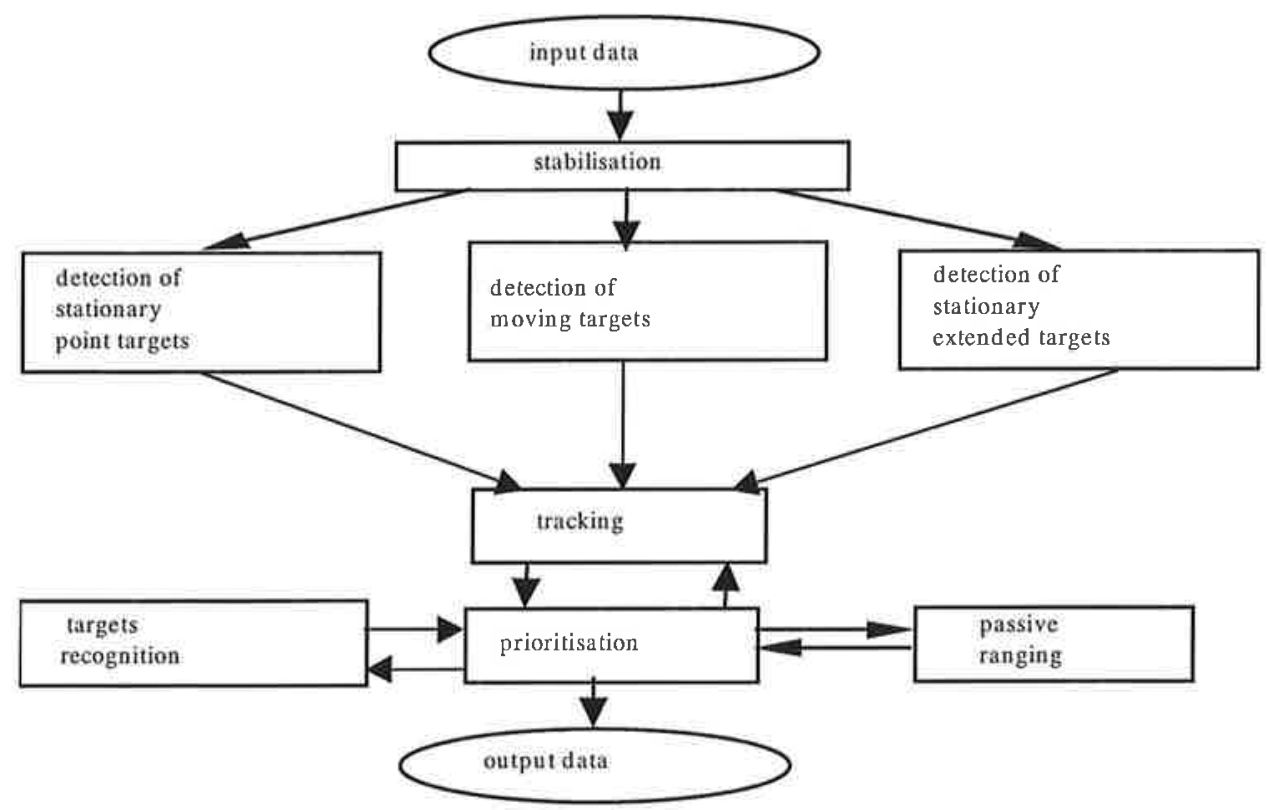

Figure 9: Algorithm combination diagram

\section{SIMULATOR IMPLEMENTATION}




\subsection{Framework (FW)}

As the simulator is built by assembling SW developed by different partners (belonging to different nations), the integration of a large amount of programs could be a difficult task if the merging work were not helped by a suitable tool. For this the use of a FW has been requested from the very beginning of the project.

The main requirements of the FW are:

- it must allow an easy integration of software developed even with different programming languages

- it must provide tools for data processing

- it must allow a graphical visualisation of all the system stages and of their connections (interactive data flow display)

- it must provide facilities for image sequences manipulation:

- it must provide tools for presentation of the results: 2-3 D plots.

In order to select the FW, many commercially available integrated SW packages have been investigated for their ability to set-up and develop the simulator as well as for performing the essential simulator's functionality's.

The compared products were $8 \mathrm{SW}$ packages: AVS/Express, IBM Data Explorer, ALADIN, IDL, IRIS Explorer, Khoros, Matlab, PV-WAVE. Comparisons concerned the following main items: platform (on which it is possible to install the SW package), architecture, general and functional requirements, technical requirements, connectivity, support from the vendor and cost. At the end Khoros package has been chosen as FW to develop the simulator.

The Khoros environment integrates multiple user interface modules, code generators, instructional aids, data visualisation, distributed computing and information processing. It consists of six major subsystems (toolboxes) which allow an easy tailoring to target specific application domain and processing environments.

\subsection{Simulator tools}

The selected FW (Khoros) provides all the tools to build every Simulator's function: if a needed function is not present in the FW it will be added.

The simulator data flow is performed using Khoros workspaces and procedures. Khoros is particularly suited to manage image processing chains (this is a major reason of the preference of Khoros). Inside the high level visual programming environment (Cantata) graphical procedures are available to support the development of hierarchical data flow graph. They are like subroutines in the textual programming language and allow to modularise the visual program.

The simulator analytical model is carried out using variable and expressions, that is Khoros tools to define variables and evaluate expressions.

Master forms, subforms and panes constitute the hierarchical GUI (Graphical User Interface) provided by the FW to select procedures inside the same flow chain. This assists the user in categorising the procedures in classes and subclasses. The panes are also used for inserting input parameters.

The file selection is performed using file/directory browsers. The alias browser allows to select the filename by means of its alias (shorthand for long pathnames). So It allows to conceptually group input files (of the START database) depending on the type of background, targets, etc.

Parameters and data input are performed by using GUI and ASCII files.

Interactive results presentation tools include image display and manipulation packages, 2D/3D plotting package.

The on-line help pages are accessible by the user through an help button on the user interface: a hierarchical organisation of the help information corresponds to the hierarchical organisation of the user interface.

\section{CONCLUSIONS}

In the EUCLID RTP 8.2 program, new IRST system concepts have been proposed. A software simulator which allows the performance comparison with other system concepts and technologies have been conceived. The simulator is based on two parallel data flow: a partial image and a performance chain. Links between the two approaches are performed through empirical rules (RoT) obtained from statistical evaluation of the IRST subsystems behaviour. The simulator structure allows also the insertion of results coming from external technology test beds. Each simulator main module has been described, and the SW implementation which uses Khoros as framework, has been outlined. 
This work was carried out in the EUCLID Programme, we are very grateful to the other consortium partners (CEALETI, CRL, INETI, Pilkington Optronics, SOFRADIR,) for their support.

\section{REFERENCES}

1. S. de Vito et al., "RTP 8.2: Intelligent sensors-New technology and design concepts for Infrared Search and Track systems", in: Proceedings "2nd NATO-IRIS Joint Conference, London 25-28 June, 1996", vol. 41, no.4, pp.31 (IV)

2. Y.Itakura, S. Tsutsumi, T. Takagi, "Statistical properties of the background noise for the atmospheric windows in the intermediate IR regions", Infrared Physics, vol. 14, n. 10, 1974

3. M. I. Smith, D. Hickman, D. Murray-Smith" Mathematical modelling of electro-optic system using a generic modelling environment”, SPIE Conference Orlando Fl., April 1997 